\title{
Heat, Resolvent and Wave Kernels with Multiple Inverse Square Potential on the Euclidian Space $\mathbb{R}^{n}$
}

\section{Mohamed Vall Ould Moustapha}

Unité de Recherche: Analyse, EDP et Modélisation (AEDPM), Département de Mathématiques et Informatique, Faculté des Sciences et Techniques, Université des Sciences, de Technologie et de la Médecine (USTM), Nouakchott, Mauritanie

Email: khames@ustm.mr

Received 26 June 2014; revised 30 July 2014; accepted 8 August 2014

Copyright @ 2014 by author and Scientific Research Publishing Inc.

This work is licensed under the Creative Commons Attribution International License (CC BY).

http://creativecommons.org/licenses/by/4.0/

c) (i) Open Access

\section{Abstract}

In this paper, the heat, resolvent and wave kernels associated to the Schrödinger operator with multi-inverse square potential on the Euclidian space $\mathbb{R}^{n}$ are given in explicit forms.

\section{Keywords}

Heat Kernel, Wave Kernel, Resolvent Kernel, Multiple-Inverse Square Potential, Bessel Function, Lauricella Hypergeometric Function

\section{Introduction}

This article is devoted to the explicit formulas for the Schwartz integral kernels of the heat, resolvent and wave operators $\mathrm{e}^{t \Delta_{v}},\left(\Delta_{v}+\lambda^{2}\right)^{-1}$ and $\cos t \sqrt{-\Delta_{v}}$ attached to the Schrödinger operator with Multiple-inverse square potential on the Euclidian space $\mathbb{R}^{n}$ :

$$
\Delta_{v}=\sum_{j=1}^{n} \frac{\partial^{2}}{\partial x_{j}^{2}}+\sum_{j=1}^{n} \frac{1 / 4-v_{j}^{2}}{x_{j}^{2}}
$$

where $v=\left(v_{1}, v_{2}, \cdots, v_{n}\right) \in \mathbb{R}^{n}$.

Note that the Schrödinger operator with bi-inverse square potential in the Euclidian plane is considered in Boyer [1] and Ould Moustapha [2]. 
For future use we recall the following formulas for the modified Bessel function of the first kind $I_{v}$ and the Hankel function of the first kind $H_{v}^{(1)}$.

$$
I_{v}(z)=\frac{(2 z)^{v} \mathrm{e}^{z}}{\sqrt{\pi} \Gamma(v+1 / 2)} \int_{0}^{1} \mathrm{e}^{-2 z t}[t(1-t)]^{\nu-1 / 2} \mathrm{~d} t
$$

(see Temme [3], p. 237).

$$
I_{v}(x) \sim \frac{(x / 2)^{v}}{\Gamma(v+1)} \quad x \rightarrow 0 \quad v \neq-1,-2, \cdots
$$

(see Temme [3], p. 234).

$$
I_{v}(x) \sim \mathrm{e}^{x}(2 \pi x)^{-1 / 2} \quad x \rightarrow \infty
$$

(see Temme [3], p. 240).

$$
H_{v}^{(1)}(z)=\frac{2}{i \sqrt{\pi} \Gamma(1 / 2-v)}(z / 2)^{-v} \int_{1}^{\infty} \mathrm{e}^{i z t}\left(t^{2}-1\right)^{-v-1 / 2} \mathrm{~d} t
$$

(see Erdély et al. [4], p. 83).

$$
H_{v}^{(1)}\left(z \sqrt{\alpha^{2}}\right)=\frac{-i}{\pi} \mathrm{e}^{-i v \pi / 2}\left(\alpha^{2}\right)^{v / 2} \int_{0}^{\infty} \mathrm{e}^{i \frac{z}{2}\left(t+\frac{\alpha^{2}}{t}\right)} t^{-v-1} \mathrm{~d} t
$$

$\operatorname{Im} z>0$ and $\operatorname{Im} \alpha^{2} z>0$ (see Magnus et al. [5], p. 84). Recall also that the $n$ variables Lauricella hypergeometric function $F_{A}^{(n)}(\alpha, \beta, \gamma, z)$ is given by (see Appell et al. [6], p. 114)

$$
F_{A}^{(n)}(\alpha, \beta, \gamma, z)=\sum_{m 0} \frac{(\alpha)_{|m|}(\beta)_{m}}{(\gamma)_{m} m !} z^{m}
$$

where $m=\left(m_{1}, m_{2}, \cdots, m_{n}\right) \in \mathbb{N}^{n}, \quad|m|=m_{1}+m_{2}+\cdots+m_{n}, \quad \alpha \in \mathbb{C} ; \quad \beta=\left(\beta_{1}, \beta_{2}, \cdots, \beta_{n}\right), \quad \gamma=\left(\gamma_{1}, \gamma_{2}, \cdots, \gamma_{n}\right)$ and $z=\left(z_{1}, z_{2}, \cdots, z_{n}\right)$ are in $R^{n}$. In the sekel we use the integral representation (see Appell et al. [6], p. 115)

$$
F_{A}^{(n)}(\alpha, \beta, \gamma, z)=c \int_{0}^{1} \int_{0}^{1} \cdots \int_{0}^{1} \prod_{j=1}^{j=n}\left(1-u_{j}\right)^{\gamma_{j}-\beta_{j}-1} u_{j}^{\beta_{j}-1}\left(1-\sum_{j=1}^{j=n} u_{j} z_{j}\right)^{-\alpha} \mathrm{d} u_{j}
$$

where

$$
c=\frac{\prod_{j=1}^{n} \Gamma\left(\gamma_{j}\right)}{\prod_{j=1}^{n} \Gamma\left(\beta_{j}\right) \Gamma\left(\gamma_{j}-\beta_{j}\right)} .
$$

For $n=2$ we have

$$
F_{A}^{(2)}(\alpha, \beta, \gamma, z)=F_{2}\left(\alpha, \beta_{1}, \beta_{2}, \gamma_{1}, \gamma_{2}, z_{1}, z_{2}\right)
$$

where $F_{2}$ is the Apple hyprgeometric function of two variables.

Recall also the following formulas for the heat kernel associated to the Schrödinger operator with inverse square potential $L_{v}=\frac{\partial^{2}}{\partial x^{2}}+\frac{1 / 4-v^{2}}{x^{2}}$.

(see Calin et al. [7], p. 68).

$$
\mathrm{e}^{t L_{v}}=\frac{\left(x x^{\prime}\right)^{1 / 2}}{2 t} \mathrm{e}^{\frac{-\left(x^{2}+x^{\prime 2}\right)}{4 t}} I_{v}\left(\frac{x x^{\prime}}{2 t}\right)
$$

where $I_{v}$ is the modified Bessel function of the first kind. 
Proposition 1.1. The Schwartz integral kernel of the heat operator with multiple-inverse square potential $\mathrm{e}^{t \Delta_{v}}$ can be written for $p=\left(x_{1}, x_{2}, \cdots, x_{n}\right), \quad p^{\prime}=\left(x_{1}^{\prime}, x_{2}^{\prime}, \cdots, x_{n}^{\prime}\right) \in \mathbb{R}_{+}^{n}$ and $t \in \mathbb{R}_{+}$as

$$
H_{v}\left(t, p, p^{\prime}\right)=\prod_{j=1}^{n} \frac{\left(x_{j} x_{j}^{\prime}\right)^{1 / 2}}{2 t} \mathrm{e}^{-\left(x_{j}^{2}+x_{j}^{\prime 2}\right) / 4 t} I_{v_{j}}\left(x_{j} x_{j}^{\prime} / 2 t\right)
$$

where $I_{v_{j}}$ is the modified Bessel function of the first kind and of order $v_{j}$.

Proof. The Formula (1.12) is a direct consequence of the Formula (1.11) and the properties of the operator (1.1).

\section{Resolvent Kernel with Multiple-Inverse Square Potential on the Euclidian Space $\mathbb{R}^{n}$}

Theorem 2.1. The Schwartz integral kernel for the resolvent operator $\left(\Delta_{v}+\lambda^{2}\right)^{-1}$ is given by the formula.

$$
\begin{aligned}
G_{v}\left(\lambda, p, p^{\prime}\right)= & c_{1} \prod_{j=1}^{n}\left(x_{j} x_{j}^{\prime}\right)^{1 / 2+v_{j}} \int_{0}^{1} \int_{0}^{1} \cdots \int_{0}^{1}\left(\frac{\lambda}{\sqrt{\left|p-p^{\prime}\right|^{2}+4 \sum_{j=1}^{n} x_{j} x_{j}^{\prime} u_{j}}}\right)^{|v|+n-1} \\
& \times H_{|v|+n-1}^{(1)}\left(\lambda \sqrt{\left|p-p^{\prime}\right|^{2}+4 \sum_{j=1}^{n} x_{j} x_{j}^{\prime} u_{j}}\right) \prod_{j=1}^{n}\left[u_{j}\left(1-u_{j}\right)\right]^{v_{j}-1 / 2} \mathrm{~d} u_{j}
\end{aligned}
$$

where $H_{v}^{(1)}$ is the Hankel function of the first kind and $c_{1}=\frac{2^{|v|-1} \mathrm{e}^{i(n-1+|v|) \pi / 2}}{i^{n+|v|-2} \pi^{(n-2) / 2} \prod_{j=1}^{n} \Gamma\left(v_{j}+1 / 2\right)}$.

Proof. We use the well known formula connecting the resolvent and the heat kernels:

$$
G_{v}\left(\lambda, p, p^{\prime}\right)=\int_{0}^{\infty} \mathrm{e}^{\lambda^{2} t} H_{v}\left(t, p ; p^{\prime}\right) \mathrm{d} t ; \quad \mathcal{R} e \lambda^{2}<0 .
$$

We combine the Formulas (2.2), (1.12) and (1.2) then use the Formulas (1.3) and (1.4) to appley the Fubini theorem and in view of the Formula (1.6) we get the Formula (2.1) and the proof of the Theorem 2.1 is finished.

Theorem 2.2. The Schwartz integral kernel of the resolvent operator $\left(\Delta_{v}+\lambda^{2}\right)^{-1}$ can be written as

$$
G_{v}\left(\lambda, p, p^{\prime}\right)=c_{2} \prod_{j=1}^{n}\left(x_{j} x_{j}^{\prime}\right)^{1 / 2+v_{j}} \int_{\left|p-p^{\prime}\right|}^{\infty} \mathrm{e}^{\mathrm{i} \lambda s}\left(s^{2}-\left|p-p^{\prime}\right|^{2}\right)^{-\alpha} F_{A}^{(n)}(\alpha, \beta, 2 \beta, z) \mathrm{d} s
$$

with $\alpha=n-1 / 2+|v|, \quad \beta_{j}=1 / 2+v_{j}, \quad z_{j}=\frac{4 x_{j} x_{j}^{\prime}}{s^{2}-\left|p-p^{\prime}\right|^{2}} j=1, \cdots, n$ and

$$
c_{2}=\frac{2^{n+2|v|-1} \mathrm{e}^{i(n-1+|v|) \pi / 2}}{\pi^{(n-1) / 2} \Gamma(3 / 2-n-|v|) i^{n+|v|-1}} \prod_{j=1}^{n}\left(\frac{\Gamma\left(1 / 2+v_{j}\right)}{\Gamma(2 v+1)}\right)
$$

where $F_{A}^{(n)}(\alpha, \beta, 2 \beta, z)$ is the n-variables Lauricella hypergeometric function given in (1.7).

Proof. We use the Formulas (2.1) and (1.5) as well as the Fubini theorem to arrive at the announced Formula (2.3).

\section{Wave Kernel with Multiple-Inverse Square Potential on the Euclidian Space}

It is known that the energy and information can only be transmitted with finite speed, smaller or equal to the speed of light. The mathematical framework, which allows an analysis and proof of this phenomenon, is the theory the wave equation. The result, which may be obtained, runs under the name finite propagation speed (see Cheeger et al. [8]). The following theorem illustrates the principle of the finite propagation speed in the case of the Schrödinger operator with multiple-inverse square potential. 
Theorem 3.1. (Finite propagation speed) Let $W_{v}\left(t, p, p^{\prime}\right)$ be the Schwartz integral kernel of the wave operator $\frac{\sin t \sqrt{-\Delta_{v}}}{\sqrt{-\Delta_{v}}}$, then we have

$$
W_{v}\left(t, p, p^{\prime}\right)=0 \quad \text { whenever }\left|p-p^{\prime}\right|>t .
$$

Proof. The proof of this result use an argument of analytic continuation from the identity

$$
\frac{\sin t \sqrt{-\Delta_{v}}}{\sqrt{-\Delta_{v}}}=\frac{1}{2 i}\left(\frac{\mathrm{e}^{i t \sqrt{-\Delta_{v}}}}{\sqrt{-\Delta_{v}}}-\frac{\mathrm{e}^{-i t \sqrt{-\Delta_{v}}}}{\sqrt{-\Delta_{v}}}\right) \text {. }
$$

We recall the formula [9], p. 50

$$
\frac{\mathrm{e}^{-t \lambda}}{t}=\frac{1}{\sqrt{\pi}} \int_{0}^{\infty} \mathrm{e}^{-u t^{2}} u^{-1 / 2} \mathrm{e}^{-\lambda^{2} / 4 u} \mathrm{~d} u
$$

By setting $t=\sqrt{-\Delta_{v}}$ and $\lambda=s$ in (3.3) we can write

$$
\mathrm{e}^{-s \sqrt{-\Delta_{v}}}=\frac{1}{\sqrt{\pi}} \int_{0}^{\infty} \mathrm{e}^{-s^{2} / 4 u} u^{-1 / 2} \mathrm{e}^{u \Delta_{v}} \mathrm{~d} u
$$

and let $P_{v}\left(s, p, p^{\prime}\right)$ be the integral kernel of $\mathrm{e}^{-s \sqrt{-\Delta_{v}}}$ then we can write

$$
P_{v}\left(s, p, p^{\prime}\right)=\frac{1}{\sqrt{\pi}} \int_{0}^{\infty} \mathrm{e}^{-s^{2} / 4 z} z^{-1 / 2} H_{v}\left(z, p, p^{\prime}\right) \mathrm{d} z
$$

where $H_{v}\left(z, p, p^{\prime}\right)$ is the heat kernel with the multiple-inverse square potential given by (1.12).

Consider the integral

$$
J(\tau)=P_{v}\left(\tau, p, p^{\prime}\right)=\frac{1}{\sqrt{\pi}} \int_{0}^{\infty}\left|\mathrm{e}^{-\tau^{2} / 4 z} z^{-1 / 2} H_{v}\left(z, p, p^{\prime}\right)\right| \mathrm{d} z
$$

using (1.12) we have

$$
J(\tau)=\frac{\prod_{j=1}^{n}\left(x_{j} x_{j}^{\prime}\right)^{1 / 2}}{\sqrt{\pi} 2^{n}} \int_{0}^{\infty}\left|\mathrm{e}^{-\tau^{2} / 4 s} s^{-1 / 2-n} \mathrm{e}^{-\left(|p|^{2}+\left|p^{\prime}\right|^{2}\right) / 4 s} \prod_{j=1}^{n} I_{v_{j}}\left(\left(x_{j} x_{j}^{\prime}\right) / 2 s\right)\right| \mathrm{d} s .
$$

From (3.2) we have

$$
W_{v}\left(t, x, x^{\prime}\right)=\frac{1}{2 i}\left(P_{v}\left(p, p^{\prime}, i t\right)-P_{v}\left(p, p^{\prime},-i t\right)\right)=\frac{1}{2 i}(J(i t)-J(-i t)) .
$$

Now set

$$
J(\tau)=J_{1}(\tau)+J_{2}(\tau)
$$

where

$$
\begin{aligned}
& J_{1}(\tau)=\frac{\prod_{j=1}^{n}\left(x_{j} x_{j}^{\prime}\right)^{1 / 2}}{\sqrt{\pi} 2^{n}} \int_{0}^{1}\left|\mathrm{e}^{-\tau^{2} / 4 s} s^{-1 / 2-n} \mathrm{e}^{-\left(|p|^{2}+\left|p^{\prime}\right|^{2}\right) / 4 s} \prod_{j=1}^{n} I_{v_{j}}\left(\left(x_{j} x_{j}^{\prime}\right) / 2 s\right)\right| \mathrm{d} s \\
& J_{2}(\tau)=\frac{\prod_{j=1}^{n}\left(x_{j} x_{j}^{\prime}\right)^{1 / 2}}{\sqrt{\pi} 2^{n}} \int_{1}^{\infty}\left|\mathrm{e}^{-\tau^{2} / 4 s} s^{-1 / 2-n} \mathrm{e}^{-\left(|p|^{2}+\left|p^{\prime}\right|^{2}\right) / 4 s} \prod_{j=1}^{n} I_{v_{j}}\left(\left(x_{j} x_{j}^{\prime}\right) / 2 s\right)\right| \mathrm{d} s .
\end{aligned}
$$

Using the Formula (1.3) we see that the last integral $J_{2}(\tau)$ converge absolutely and is analytic in $\tau$ for $n-1 / 2+|v|>0$. 
For the first integral $J_{1}(\tau)$ we obtain

$$
J_{1}(\tau)=\frac{\prod_{j=1}^{n}\left(x_{j} x_{j}^{\prime}\right)^{1 / 2}}{\sqrt{\pi} 2^{n}} \int_{1}^{\infty}\left|\mathrm{e}^{-\tau^{2} y / 4} y^{-3 / 2+n} \mathrm{e}^{-\left(|p|^{2}+\left|p^{\prime}\right|^{2}\right) y / 4} \prod_{j=1}^{n} I_{v_{j}}\left(\left(x_{j} x_{j}^{\prime}\right) y / 2\right)\right| \mathrm{d} y
$$

and from the Formula (1.4) we see that

$$
J_{1}(\tau)=\frac{\prod_{j=1}^{n}\left(x_{j} x_{j}^{\prime}\right)^{1 / 2}}{\sqrt{\pi} 2^{n}} \int_{1}^{\infty}\left|\mathrm{e}^{-y\left(\left(\mid p-p^{\prime 2}\right) / 4+\tau^{2} / 4\right)} y^{-3 / 2+n+|v|}\right| \mathrm{d} y
$$

is analytic in $\tau$ and converge if $\mathcal{R} e\left[\tau^{2}+\left|p-p^{\prime}\right|^{2}\right]>0$, hence the integral $J( \pm i t)$ is absolutely convergent if $( \pm i t)^{2}+\left|p-p^{\prime}\right|^{2}>0$ (i.e.) $\left|p-p^{\prime}\right|>t$ and in view of (3.8) we have $W_{v}\left(t, p, p^{\prime}\right)=0$ for $\left|p-p^{\prime}\right|>t$ and the proof of the Theorem 3.1 is finished.

Theorem 3.2. The Schwartz integral kernel for the wave operator $\cos t \sqrt{-\Delta_{v}}$ with multiple-inverse square potential on the Euclidian space can be written on the two following forms

$$
w_{v}\left(t, p, p^{\prime}\right)=\frac{\prod_{j=1}^{n}\left(x_{j} x_{j}^{\prime}\right)^{1 / 2}}{2 i \sqrt{2 \pi} t^{2 n}} \int_{-\infty}^{0+} \exp \left[-\frac{u}{2 t^{2}}\left(|p|^{2}+\left|p^{\prime}\right|^{2}-t^{2}\right)\right] \prod_{j=1}^{n} I_{v_{j}}\left(\frac{x_{j} x_{j}^{\prime}}{t^{2}} u\right) u^{n-1 / 2} \mathrm{~d} u
$$

and

$$
w_{v}\left(t, p, p^{\prime}\right)=2 \frac{\prod_{j=1}^{n}\left(x_{j} x_{j}^{\prime}\right)^{1 / 2}}{\sqrt{2 \pi} t^{2 n}} \int_{0}^{\infty} \exp \left[-\frac{u}{2 t^{2}}\left(|p|^{2}+\left|p^{\prime}\right|^{2}-t^{2}\right)\right] \prod_{j=1}^{n} I_{v_{j}}\left(\frac{-x_{j} x_{j}^{\prime}}{t^{2}} u\right) u^{n-1 / 2} \mathrm{~d} u
$$

where $I_{v_{1}}$ is the first kind modified Bessel functions of order $v$.

Proof. We start by recalling the formula (see Magnus et al. [5], p. 73).

$$
\cos z=\sqrt{\pi z / 2} J_{-1 / 2}(z)
$$

where $J_{v}($.$) is the Bessel function of first kind and of order v$ defined by (see Magnus et al. [5], p. 83).

$$
J_{v}(\alpha z)=\frac{z^{v}}{2 i \pi} \int_{-\infty}^{0^{+}} \mathrm{e}^{(\alpha / 2)\left(t-z^{2} / t\right)} t^{-v-1} \mathrm{~d} t
$$

provided that $\mathcal{R} e \alpha>0$ and $|\operatorname{argz}| \leq \pi$. Here we should note that the integral in (3.17) can be extended over a contour starting at $\infty$, going clockwise around 0 , and returning back to $\infty$ without cutting the real negative semi-axis.

For $v=-1 / 2$ the Equation (3.17) can be combined with Equation (3.16) to derive the following formula.

$$
\cos \alpha z=\frac{1}{2 i \sqrt{2 \pi}} \int_{-\infty}^{0+} \mathrm{e}^{(\alpha / 2)\left(u-z^{2} / u\right)} u^{-1 / 2} \mathrm{~d} u
$$

Putting $\alpha=1$ and replacing the variable $z$ by the symbol $t \sqrt{-\Delta_{v}}$ in (3.18) we obtain

$$
\cos t \sqrt{-\Delta_{v}}=\frac{1}{2 i \sqrt{2 \pi}} \int_{-\infty}^{0+\infty} e^{\left(u / 2+\left(t^{2} / 2 u\right) \Delta_{v}\right)} u^{-1 / 2} \mathrm{~d} u .
$$

Finally making use of (1.12) in (3.19), we get the Formula (3.14).

To see the Formula (3.15) set

$$
J=\int_{-\infty}^{0+} \exp \left[-\frac{u}{2 t^{2}}\left(|p|^{2}+\left|p^{\prime}\right|^{2}-t^{2}\right)\right] \prod_{j=1}^{n} I_{v_{j}}\left(\frac{x_{j} x_{j}^{\prime}}{t^{2}} u\right) u^{n-1 / 2} \mathrm{~d} u
$$

and

$$
I=\int_{0}^{\infty} \exp \left[-\frac{u}{2 t^{2}}\left(|p|^{2}+\left|p^{\prime}\right|^{2}-t^{2}\right)\right] \prod_{j=1}^{n} I_{v_{j}}\left(\frac{x_{j} x_{j}^{\prime}}{t^{2}} u\right) u^{n-1 / 2} \mathrm{~d} u
$$


we have

$$
\begin{gathered}
J=J_{1}+J_{2}+J_{3} \\
J_{1}=\int_{\gamma_{1}} \exp \left[-\frac{u}{2 t^{2}}\left(|p|^{2}+\left|p^{\prime}\right|^{2}-t^{2}\right)\right] \prod_{j=1}^{n} I_{v_{j}}\left(\frac{x_{j} x_{j}^{\prime}}{t^{2}} u\right) u^{n-1 / 2} \mathrm{~d} u \\
J_{2}=\int_{\gamma_{2}} \exp \left[-\frac{u}{2 t^{2}}\left(|p|^{2}+\left|p^{\prime}\right|^{2}-t^{2}\right)\right] \prod_{j=1}^{n} I_{v_{j}}\left(\frac{x_{j} x_{j}^{\prime}}{t^{2}} u\right) u^{n-1 / 2} \mathrm{~d} u
\end{gathered}
$$

and

$$
J_{3}=\int_{\gamma_{3}} \exp \left[-\frac{u}{2 t^{2}}\left(|p|^{2}+\left|p^{\prime}\right|^{2}-t^{2}\right)\right] \prod_{j=1}^{n} I_{v_{j}}\left(\frac{x_{j} x_{j}^{\prime}}{t^{2}} u\right) u^{n-1 / 2} \mathrm{~d} u
$$

where the paths $\gamma_{1}, \gamma_{2}$ and $\gamma_{3}$ are given by

$$
\begin{gathered}
\gamma_{1}: z=r \mathrm{e}^{i \pi} ; \epsilon \leq r<\infty \text { (above the cut) } \\
\gamma_{2}: z=r \mathrm{e}^{-i \pi} ; \infty>r \geq \epsilon \text { (below the cut) } \\
\gamma_{3}: z=\epsilon \mathrm{e}^{i \theta} ;-\pi<\theta<\pi \text { (rund the small circle) }
\end{gathered}
$$

as $\epsilon \rightarrow 0$, we have $J_{1} \rightarrow \mathrm{e}^{(n+1 / 2) i \pi} I, \quad J_{2} \rightarrow \mathrm{e}^{-(n+1 / 2) i \pi} I$ and $J_{3} \rightarrow 0$.

Adding the integrals establishes the required results $J=2 i \sin (n+1 / 2) \pi I$.

Theorem 3.3. The integral kernel for the wave operator $\cos t \sqrt{-\Delta_{v}}$ with multiple-inverse square potential on the Euclidian plane can be written as

$$
w_{v}\left(t, p, p^{\prime}\right)=c_{3} \prod_{j=1}^{n}\left(x_{j} x_{j}^{\prime}\right)^{v_{j}+1 / 2} t\left(t^{2}-\left|p-p^{\prime}\right|^{2}\right)_{+}^{-\alpha} F_{A}^{(n)}(\alpha, \beta, 2 \beta, z)
$$

where $F_{A}^{(n)}(\alpha, \beta, \gamma, z)$ is the $n$-variables Lauricella hypergeometric function $F_{A}^{(n)}$ given in (1.7), $\alpha=n+1 / 2+|v|, \quad \beta=\left(\beta_{1}, \beta_{2}, \cdots, \beta_{n}\right), \quad \beta_{j}=v_{j}+1 / 2 \quad$ and $z_{j}=4 x_{j} x_{j}^{\prime} /\left(t^{2}-\left|p-p^{\prime}\right|^{2}\right) 1 \leq j \leq n$ and the constant $c_{j}$ is given by

$$
c_{3}=\frac{(-1)^{|v|} 2^{n+1+2|v|} \Gamma(n+1 / 2+|v|)}{\pi^{(n+1) / 2}} \prod_{j=1}^{n} \frac{\Gamma\left(1 / 2+v_{j}\right)}{\Gamma\left(2 v_{j}+1\right)} .
$$

Proof. We use essetially the Formula (3.15) of Theorem 3.2, the Formulas (1.2), the Fubini theorem and the Formula (1.8).

\section{Applications and Further Studies}

We give an application of the Theorem 3.3.

Corollary 4.1. The integral kernel of the heat operator $\mathrm{e}^{t \Delta_{v}}$ can be written in the form

$$
H_{v}\left(t, p, p^{\prime}\right)=\frac{c_{4}}{\sqrt{t}} \prod_{j=1}^{n}\left(x_{j} x_{j}^{\prime}\right)^{v_{j}+1 / 2} \int_{\left|p-p^{\prime}\right|}^{\infty} \mathrm{e}^{-u^{2} / 4 t} u\left(u^{2}-\left|p-p^{\prime}\right|^{2}\right)^{-\alpha} F_{A}^{(n)}(\alpha, \beta, 2 \beta, z) \mathrm{d} u
$$

with $\alpha=n+1 / 2+|v|, \quad \beta_{j}=v_{j}+1 / 2$ and $z_{j}=4 x_{j} x_{j}^{\prime} /\left(u^{2}-\left|p-p^{\prime}\right|^{2}\right), \quad 1 \leq j \leq n$ and

$$
c_{4}=\frac{(-1)^{|v|} 2^{n+1+2|v|} \Gamma(n+1 / 2+|v|)}{\pi^{n / 2+1}} \prod_{j=1}^{n} \frac{\Gamma\left(1 / 2+v_{j}\right)}{\Gamma\left(2 v_{j}+1\right)} .
$$

Proof. We use the transmutation formula (see Greiner et al. [10], p. 362) 


$$
\mathrm{e}^{t \Delta_{v}}=\frac{1}{\sqrt{\pi t}} \int_{0}^{\infty} \mathrm{e}^{-u^{2} / 4 t} \cos u \sqrt{-\Delta_{v}} \mathrm{~d} u .
$$

We suggest here a certain number of open related problems connected to this paper. For example the semilinear wave and heat equations associated to the multiple-inverse square potential and its global solution and a possible blow up of the solution in finite times.

We can also to look for the dispersive and Strichartz estimates for the Schrödinger and the wave equations with multiple-inverse square potential, for the case of inverse square potential (Burg et al. [11] and Planchon et al. [12]).

\section{References}

[1] Boyer, C.P. (1976) Lie Theory and Separation of Variables for the Equation $i U_{t}+\left(\frac{\alpha}{x_{1}^{2}}+\frac{\beta}{x_{2}^{2}}\right) U=0$. SIAM Journal on Mathematical Analysis, 7, 230-263. http://dx.doi.org/10.1137/0507019

[2] Ould Moustapha, M.V. (2014) The Heat, Resolvent and Wave Kernels with Bi-Inverse Square Potential on the Euclidean Plane. International Journal of Applied Mathematics, 27, 127-136.

[3] Temme, N.M. (1996) Special Functions: An Introduction to the Classical Functions of Mathematical Physics. John Wiley and Sons, Inc., New-York.

[4] Erdélyi, A., Magnus, W., Oberhettinger, F. and Tricomi, F.G. (1954) Transcendental Functions, Tome II. New York.

[5] Magnus, W., Oberhettinger, F. and Soni, R.P. (1966) Formulas and Theorems for the Special Functions of Mathematical Physics. Springer-Verlag, New York. http://dx.doi.org/10.1007/978-3-662-11761-3

[6] Appell, P. and Kampe de Feriet, M.J. (1926) Fonctions Hypergeometriques et Hyperspheriques. Polynôme d’Hermite. Gauthier-Villars, Paris.

[7] Calin, O., Chang, D., Furutani, K. and Iwasaki, C. (2011) Heat Kernels for Elliptic and Sub-Elliptic Operators Methods and Techniques. Springer, New York. http://dx.doi.org/10.1007/978-0-8176-4995-1

[8] Cheeger, J. and Taylor, M. (1982) On the Diffraction of Waves by Canonical Singularites I. Communications on Pure and Applied Mathematics, 35, 275-331. http://dx.doi.org/10.1002/cpa.3160350302

[9] Strichartz, R. (2003) A Guide to Distribution Theory and Fourier Transform, Studies in Advanced Mathematics. CRC Press, Boca Racon.

[10] Greiner, P.C., Holocman, D. and Kannai, Y. (2002) Wave Kernels Related to the Second Order Operator. Duke Mathematical Journal, 114, 329-387. http://dx.doi.org/10.1215/S0012-7094-02-11426-4

[11] Burg, N., Planchon, F., Stalker, J. and Tahvildar-Zadeh, A.S. (2002) Strichartz Estimate for the Wave Equation with the Inverse Square Potential. arXiv:math.AP/0207152v3.

[12] Planchon, F., Stalker, J. and Tahvildar-Zadeh, A.S. (2003) Dispersive Estimate for the Wave Equation with the Inverse Square Potential. Discrete and Continuous Dynamical Systems, 9, 1337-1400. 
Scientific Research Publishing (SCIRP) is one of the largest Open Access journal publishers. It is currently publishing more than 200 open access, online, peer-reviewed journals covering a wide range of academic disciplines. SCIRP serves the worldwide academic communities and contributes to the progress and application of science with its publication.

Other selected journals from SCIRP are listed as below. Submit your manuscript to us via either submit@scirp.org or Online Submission Portal.
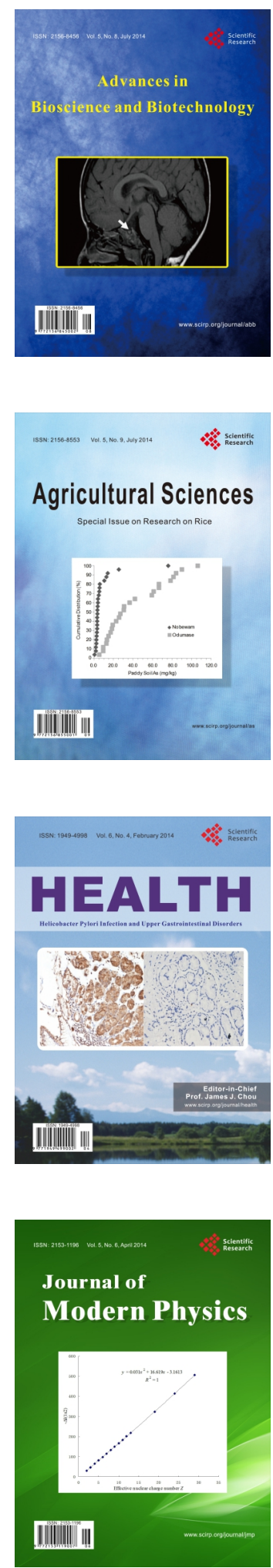
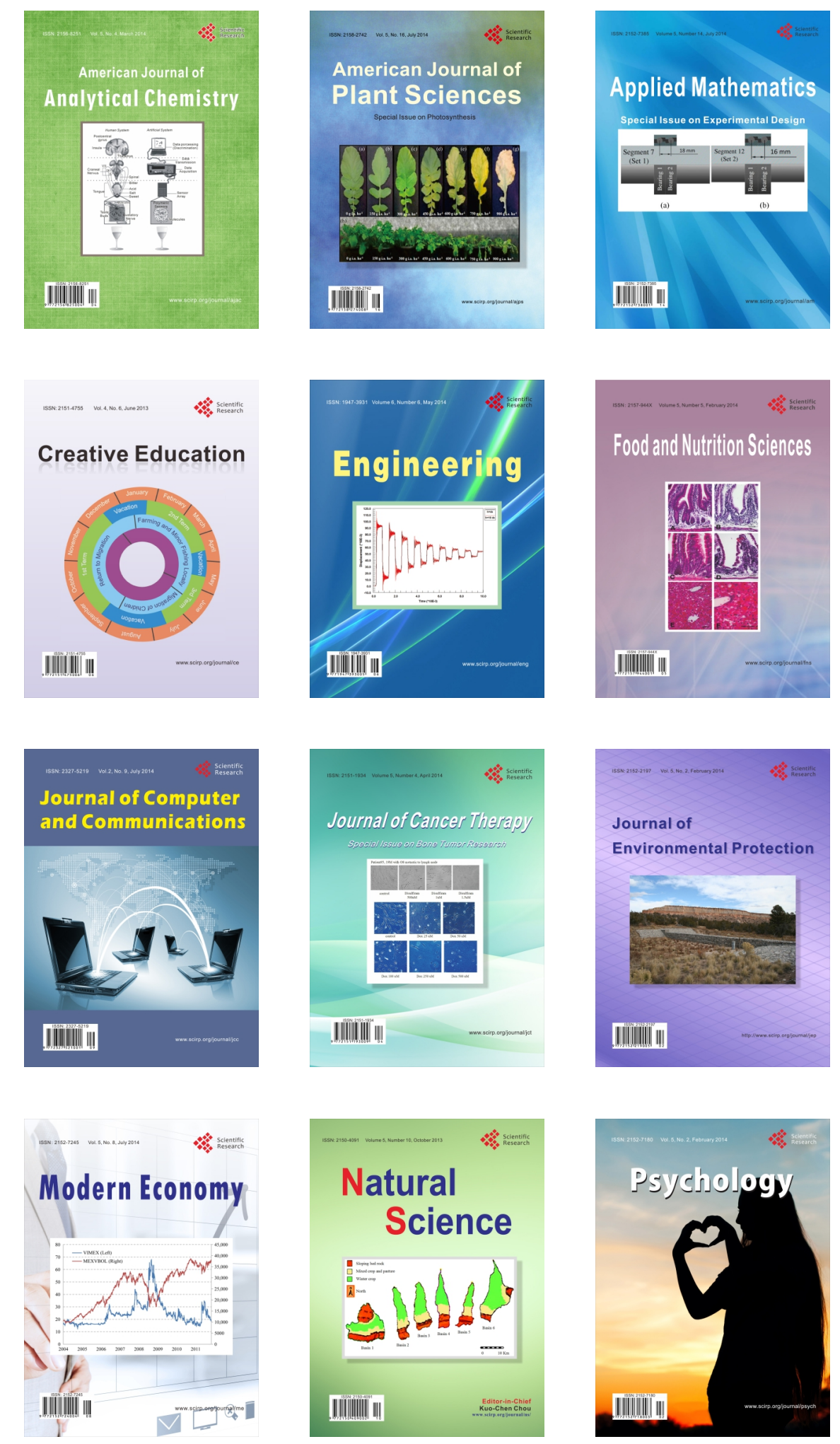